\title{
Growth Measurements on Surface Colonies of Bacteria
}

\author{
By S. A. PALUMBO,* M. G. JOHNSON, V. T. RIECK \\ AND L. D. WITTER \\ Department of Food Science, University of Illinois, Urbana, Illinois 6180 I, U.S.A. \\ (Accepted for publication I6 March 1971) \\ SUMMARY \\ Growth of colonies of Pseudomonas fluorescens on trypticase soy agar (TSA) and \\ glucose-salts agar (GSA) is described in terms of diameter, height and viable count \\ and of calculated volume and viable cell density. All increased with time except \\ viable cell density, which was constant for colonies at 3.1 I $\times 10^{8} \mathrm{cell} / \mathrm{mm}^{3}$ on TSA \\ and $10.08 \times 10^{8}$ cells $/ \mathrm{mm}^{3}$ on GSA. Colony diameter increased linearly at 0.0585 \\ $\mathrm{mm}$./h. on TSA and $0.0594 \mathrm{~mm}$./h. on GSA, but the modes of growth were very \\ different. Colonies on TSA increased in both diameter and height while those on \\ GSA increased mainly in diameter, so colony volumes on the two agars differed. \\ Colony diameters of Micrococcus caseolyticus and eight Gram-negative rods also \\ increased linearly with time. We propose that a linear increase of colony diameter \\ with time represents the steady state growth of bacterial colonies.
}

\section{INTRODUCTION}

The growth of bacterial colonies is of interest and importance in such widely divergent areas of microbiology as quality control in food processing and screening of mutants in bacterial genetics. Despite this, little is known about the growth kinetics of colonies and the effects of environment on these kinetics. By contrast, much is known about bacterial growth in broth (Monod, I949; Painter \& Marr, 1968). Growth in broth can be measured by extinction, dry weight or viable cell count, but colony growth cannot be measured by these.

Palumbo, Rieck \& Witter (1964) reported that colony diameter was a useful indicator of the steady-state growth of surface colonies. Pirt (1967) and Cooper, Dean \& Hinshelwood (1968) also used colony diameter to estimate the colony growth of Klebsiella aerogenes, Streptococcus faecalis and Escherichia coli. In this paper we have measured colony height, viable cell count and colony diameter for Pseudomonas fluorescens and colony diameter for Micrococcus caseolyticus as indices of colony growth. We also have calculated colony volume and viable cell density for colonies of $P$. fluorescens. To evaluate the influence of environmental factors, such as nutrients, we followed diameter, the only measurement that did not require the destruction of the colony.

\section{METHODS}

Organisms and temperature. A strain of Pseudomonas fuorescens, described by Sinclair \& Stokes (1962), which forms circular colonies with entire edges was incubated at $30^{\circ}$. A psychrophilic strain of Micrococcus caseolyticus from our laboratory collection was incubated at $26^{\circ}$.

Media. Pseudomonas fluorescens and Micrococcus caseolyticus were grown on trypticase

* Present address: Meat Laboratory, Eastern Utilization Research and Development Division, Agricultural Research Service, United States Department of Agriculture, Beltsville, Maryland 20705, U.S.A. 
soy agar (TSA; Baltimore Biological Laboratories). Trypticase soy broth (TSB; Baltimore Biological Laboratories) was also used for the preparation of inocula of $M$. caseolyticus. Pseudomonas fluorescens was also grown on a defined glucose-salts agar (GSA) containing $(\mathrm{w} / \mathrm{v})$ : glucose, $\mathrm{I} \% ;\left(\mathrm{NH}_{4}\right)_{2} \mathrm{SO}_{4}, 0.1 \% ; \mathrm{K}_{2} \mathrm{HPO}_{4}, 0.6 \% ; \mathrm{KH}_{2} \mathrm{PO}_{4}, 0.3 \% ; \mathrm{MgSO}_{4}, 0.02 \%$; and agar (Difco), $1.5 \%$. Inocula of $P$. fluorescens were grown in this GSA medium without agar (GSA broth). Variations of this medium, with different carbon and/or nitrogen sources, are mentioned in the Results. Different strength media were prepared by diluting and adding enough agar to restore the concentration to $\mathrm{I} \cdot 5 \%$. The medium cooled to $45^{\circ}$ (TSA or GSA) was dispensed into special flat-bottomed Petri dishes, $20 \mathrm{ml}$. for $M$. caseolyticus and $30 \mathrm{ml}$. for $P$. fluorescens (except in the volume experiments) and allowed to dry covered overnight at room temperature.

Inocula. The agar surfaces were inoculated with a $24 \mathrm{~h}$. broth culture of Micrococcus caseolyticus grown in TSB or of Pseudomonas fluorescens in GSA broth diluted in $0.1 \%$ peptone water to give three to five well isolated colonies.

Measurements. Colony diameters were measured, after removal of the covers of the dishes to avoid distortion, with a calibrated, filar-micrometer eyepiece (Bausch and Lomb) mounted on a stereoscopic microscope (Bausch and Lomb). For most experiments, ten colonies on at least five plates were measured and the average of their diameters taken and plotted against time. The $\Delta d / \Delta t$ in $\mathrm{mm}$./h. (slope) and the best fit straight line were calculated for the initial portion of the plot by the least sum of squares method.

Colony height was measured by cutting the colony and its surrounding agar from the plate, placing the plug on a clean microscope slide and supporting it so that its profile could be seen and its height measured. The profile of colonies of Pseudomonas fluorescens on TSA was photographed in this position.

After the height was measured the colony and the agar were mixed for I min. in $99 \mathrm{ml}$. peptone water in a Waring blender. Dilutions were plated on to dried TSA plates, incubated at $30^{\circ}$ and counted after $36 \mathrm{~h}$.

Calculations. By assuming the colony to be a spherical segment, its volume $(V)$ was calculated by the formula:

$$
V=\frac{1}{6} \pi h\left(3 r^{2}+h^{2}\right)
$$

where $r=\frac{1}{2}$ the measured diameter and $h=$ the measured height. For colonies of Pseudomonas fluorescens on TSA this assumption was considered reasonable in view of the photographs of the colonies' profiles. The radius $(R)$ of the sphere of which the colony is a segment can be calculated by the formula:

$$
R=\frac{\left(d^{2}+4 h^{2}\right)}{8 h}
$$

Using these radii we found that line drawings of colonies on TSA compared favourably with the pictures of the profiles.

For colonies on GSA this same assumption was made for convenience and comparison although the colonies were very much flatter than those on TSA, but the volumes of these colonies were calculated by taking them to be segments of spheres with much larger radii than the colonies on TSA.

The viable cell density of a colony was obtained by dividing the viable count per colony by the colony volume in $\mathrm{mm}^{3}$.

Growth of a colony around a barrier. Glass barriers were prepared by glueing coverslips perpendicularly on glass microscope slides with epoxy resin. During the process the cover slips were held in place by short sections of microscope slides. The resulting assemblies were 
dipped in alcohol, placed in sterile Petri dishes, and molten TSA was poured in so that only the top part of the upright sections were left uncovered. Spot inoculations of Pseudomonas fluorescens were made at specific points on the agar and the plates were incubated upright.

\section{RESULTS}

Growth studies. Ten colonies were chosen as controls. At each interval five colonies, whose diameters were similar to the average diameter of the ten controls, were cut and measured. The increase in the various parameters are presented in Fig. I to 3. In Fig. 3 growth curves for the bacteria in broths of a similar constitution as the agar are given for comparison.

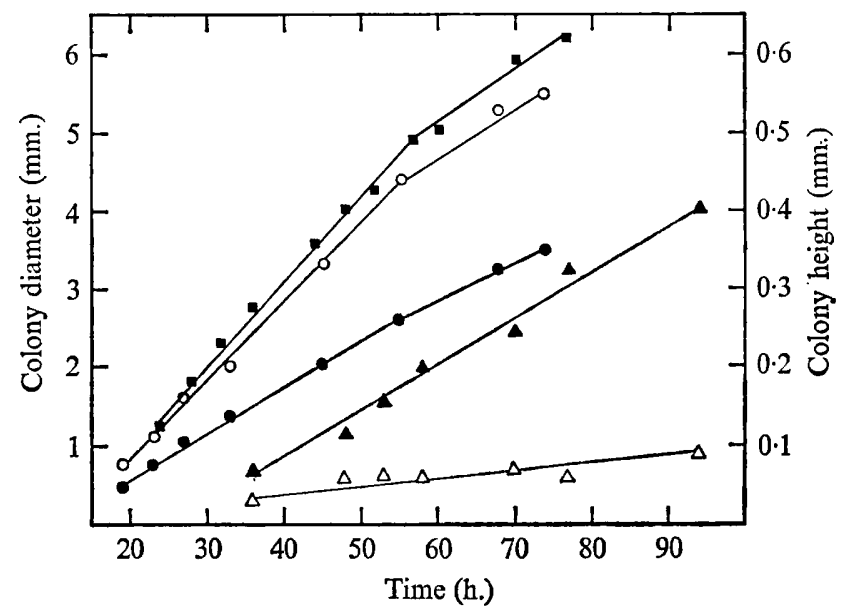

Fig. I. Effect of media on diameter and height of colonies of Micrococcus caseolyticus and Pseudomonas fluorescens (average of five colonies): diameter ( $\boldsymbol{\square})$ of $M$. caseolyticus on TSA; diameter (O) and height $(\bigcirc)$ of $P$. fluorescens on TSA; diameter $(\Delta)$ and height $(\triangle)$ of $P$. fluorescens on GSA.

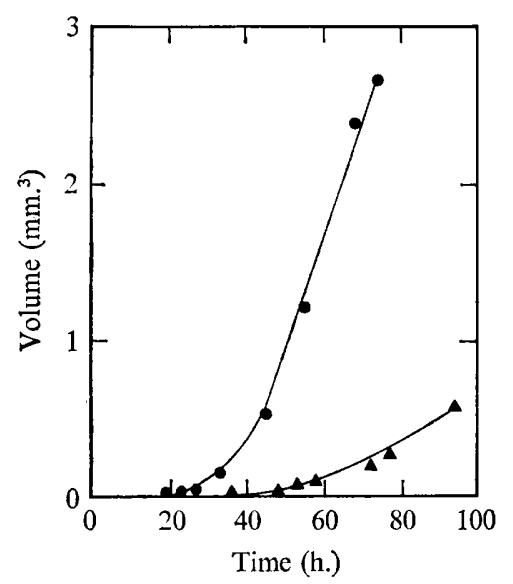

Fig. 2

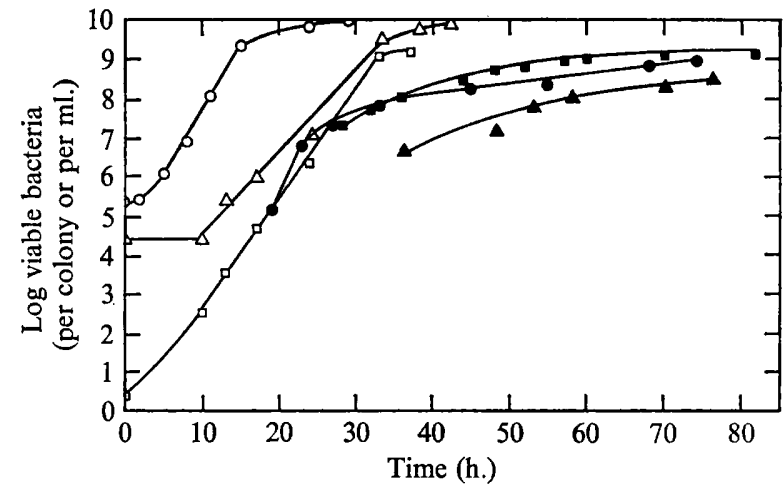

Fig. 3

Fig. 2. Effect of media on volume of colonies of Pseudomonas fluorescens (average of five colonies):

$$
\text { , TSA; } \Delta \text {, GSA. }
$$

Fig. 3. Effect of media on number of viable cells in cultures of Pseudomonas fluorescens and Micrococcus caseolyticus: $P$. fluorescens in TSA broth (O) and on TSA (O); P. fluorescens in GSA broth $(\triangle)$ and on GSA $(\Delta) ; M$. caseolyticus in TSB $(\square)$ and on TSA ( $\square)$. 
The $\Delta d / \Delta t$ for Pseudomonas fluorescens is $0.0585 \mathrm{~mm}$./h. on TSA and $0.0594 \mathrm{~mm} . / \mathrm{h}$. on GSA, while for Micrococcus caseolyticus on TSA it is $0.109 \mathrm{~mm}$./h. No other calculations of growth rate were made on colonies. Generation times could not be calculated for colonies because there were no linear portions of the plots (Fig. 3). Generation times in broth are 57 and $70 \mathrm{~min}$. respectively for $P$. fluorescens in TSA broth and GSA broth, and $75 \mathrm{~min}$. for $M$. caseolyticus in TSB. Extrapolation was made of the logarithm of the viable count per colony of $P$. fluorescens on TSA back to zero (I cell/colony) (Fig. 3) and a generation time of $45 \mathrm{~min}$. was calculated from the linear portion. Although the other plots do not extrapolate as readily, this process shows that to reach the initial measured population the organisms must have divided at a faster rate in the premeasurement period and that the growth rate was faster on agar than in broth.

Table I. Viable cell densities of colonies of Pseudomonas fluorescens on TSA and GSA

\begin{tabular}{|c|c|c|}
\hline \multirow[b]{2}{*}{ Time (h.) } & \multicolumn{2}{|c|}{ Viable cell density, } \\
\hline & TSA & GSA \\
\hline 19 & $0.3 \mathrm{I}$ & nd \\
\hline 23 & $2 \cdot 18$ & nd \\
\hline 27 & $4 \cdot 35$ & nd \\
\hline 33 & 3.54 & nd \\
\hline 36 & nd & 7.90 \\
\hline 45 & $3 \cdot 38$ & nd \\
\hline 48 & nd & $7 \cdot 39$ \\
\hline 53 & nd & II 0 \\
\hline 55 & 2.48 & nd \\
\hline 58 & nd & $12 \cdot 0$ \\
\hline 68 & $2 \cdot 84$ & nd \\
\hline 70 & nd & $\mathrm{XI} \cdot 9$ \\
\hline 74 & $2 \cdot 94$ & nd \\
\hline 77 & nd & 10.09 \\
\hline 94 & nd & $9 \cdot 48$ \\
\hline Average & $3 \cdot$ I I & 10.08 \\
\hline
\end{tabular}

Table 2. Effect of different carbon and/or nitrogen sources in the defined agar on the $\Delta d / \Delta t$ for colonies of Pseudomonas fluorescens

\begin{tabular}{lc}
\multicolumn{1}{c}{ Nutrient* } & $\Delta d / \Delta t(\mathrm{~mm} . / \mathrm{h})$. \\
Sodium lactate & 0.0683 \\
Peptone ${ }^{\dagger}$ & 0.0587 \\
Sodium glutamate $\dagger$ & 0.0572 \\
Sodium succinate & $0.044 \mathrm{I}$ \\
Glycerol & 0.0270 \\
Mannitol & 0.0249
\end{tabular}

* Added to give a I \% (w/v) concentration. $\uparrow$ As both the carbon and nitrogen source.

The viable cell densities for colonies of Pseudomonas fluorescens are given in Table I. The constancy of these values suggested that the individual measurements are accurate and reproducible and the calculations correctly chosen, and that a constant proportion of the cells in the colony is being recovered or a constant proportion are viable.

Nutrition. The influence of various nutrients and nutrient levels was evaluated by measuring the $\Delta d / \Delta t$. The $\Delta d / \Delta t$ for colonies of Pseudomonas fluorescens on GSA with other carbon 
and nitrogen sources are given in Table 2. The plots from which these $\Delta d / \Delta t$ values were obtained all showed linear increase of diameter. Decreasing the nutrient level had little or no effect on the $\Delta d / \Delta t$. The effect of various nutrients on the height of colonies was not quantitated. However, qualitative observations indicated that certain nutrients, e.g. peptone and glutamate, gave colonies similar to those on GSA, while other nutrients, e.g. glycerol and mannitol, gave colonies similar to those on TSA.

In a separate study the influence of different volumes of TSA per plate was determined. The $\Delta d / \Delta t$ for $30,20,10$ and $5 \mathrm{ml}$. of TSA was $0.0528,0.0538,0.0513$, and $0.0487 \mathrm{~mm} . / \mathrm{h}$. respectively. The diameters of these colonies also showed linear increase.

The influence of relative humidity on the growth of colonies of Micrococcus caseolyticus was studied. Two sets of TSA plates were inoculated of which one set was incubated in a water-saturated atmosphere and the other set in an air incubator at $26^{\circ}$. After $96 \mathrm{~h}$. incubation the diameters of ten colonies from each set were measured. The diameters of colonies from the two sets were similar; however, macroscopic examination of the colonies showed that those incubated in the humid atmosphere had a greater height than those incubated in the dry atmosphere.

Growth of a colony around a barrier. To test whether only the peripheral bacteria of a colony contribute to the increasing diameter the growth path of Pseudomonas fluorescens was obstructed by a barrier (Fig. 4). The organism was spot-inoculated at point 0 from which the colony grew out circularly until it met the barrier at point $\mathrm{A}$, where it grew around the barrier and re-established its circular edge. Only the bacteria at the periphery of a colony contribute to its diameter.

\section{DISCUSSION}

In this study colony growth has been estimated by measured parameters of diameter, height and viable count and calculated parameters of volume and viable cell density. All except the viable cell density increased as the colony grew, while the viable cell density was constant for a colony on a particular medium.

Despite similar $\Delta d / \Delta t$, colonies of Pseudomonas fluorescens on TSA and GSA were different. On GSA, the colonies were much flatter and had a greater viable cell density than on TSA. Pirt (1967), in his study of colony growth, concluded that the total height of a colony will not vary greatly. This holds for colonies of $P$. fluorescens on GSA but not on TSA. On nutrients such as peptone colony height was similar to that on GSA. Thus substrate, rather than oxygen, as predicted by Pirt, regulated colony height of $P$. fluorescens.

Pirt (1967) suggested that the exponential phase of growth of a colony occurred while the colony was quite small, and as soon as any nutrient became limiting the colony entered the phase of constant radial growth. None of the viable cell plots showed a linear or exponential phase. The $\Delta d / \Delta t$ apparently increased more rapidly in the premeasurement period, and probably so did the viable count.

For both Pseudomonas fluorescens and Micrococcus caseolyticus diameter increased linearly for all variables tested. Based on these data we propose that the linear increase of colony diameter with time represents the steady-state growth of bacterial surface colonies and is similar to the logarithmic growth phase in broth. Support for this proposal comes from studies of many organisms including earlier studies in our laboratory with eight strains of Gram-negative rod. Pirt (1967) and Cooper et al. (1968) observed a linear increase of colony radii and diameter for Escherichia coli, Streptococcus faecalis and Klebsiella aerogenes.

Bates (1928) studied colonies of Escherichia coli, Bacillus subtilis, B. cereus and Pseudomonas aeruginosa. Using his data and assuming that all the colonies were circular, which 
they were not, we calculated the changes in diameter and found that the diameters of these colonies increased linearly with time.

Diameter has been used to estimate the growth of fungal colonies. Trinci (1969) used radius to measure the colony growth of Aspergillus nidulans, Penicillium chrysogenum and Mucor hiemalis. Haines (1932) employed hyphal length to estimate growth of several actinomycetes and observed a linear increase of hyphal length. Emerson (1950) studied colonies of Neurospora and found that their radii increased at a constant rate over a long period of time. Diameter measurements also have been used to evaluate influence of growth variables on bacterial and fungal colonies (Stokes \& Bayne, I957; Pirt, 1967; Trinci, 1969).

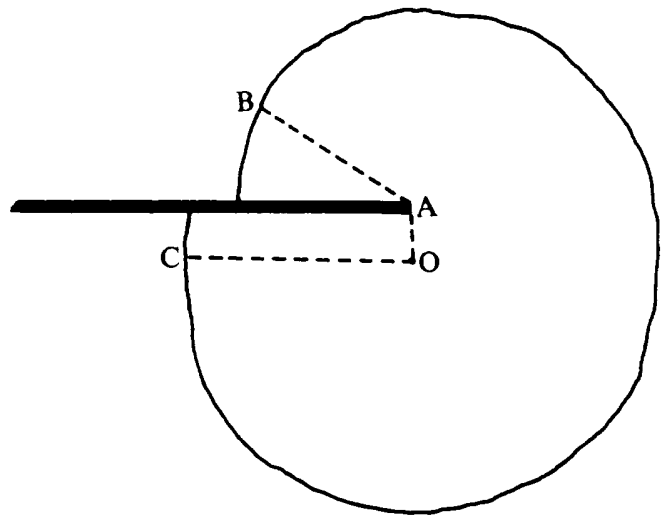

Fig. 4. Line drawing of a photomicrograph of a colony of Pseudomonas fluorescens growing around a barrier. Dark solid bar is the barrier; $\mathrm{O}$ is the point of inoculation. $\mathrm{OC}=\mathrm{AO}+\mathrm{AB}$; measurements from the figure: $\mathrm{OC}=46 \mathrm{~mm}$., $\mathrm{AB}=36 \mathrm{~mm}$., $\mathrm{OA}=\mathrm{II} \mathrm{mm}$; $46 \mathrm{~mm}$. $\cong 36 \mathrm{~mm}$. + I I mm.

A linear increase of colony diameter suggests that only the bacteria on the periphery of the colony are dividing. Support for this comes from the observed growth of the colony around the barrier (Fig. 4). Egunov (I9I5), in his study of a lactobacillus, found that each point on the periphery of the colony is a centre of growth, and that when the direction of growth is changed there is no actual loss of growth.

In addition to further data on the validity of diameter as a parameter of colony growth, we also have provided data on parameters not considered by other workers. For colonies of Pseudomonas fluorescens on TSA and GSA, we now know how many cells are in a colony, the viable cell density, and how colony diameter, height, volume and viable count vary with time.

With none of the measurements was a true exponential phase observed. Pirt's model (1967) considered the exponential phase to occur while the colony is quite small. As nutrient diffusion to the colony limits its growth, growth changes to the phase of constant radial (diameter) growth. Our data support Pirt's view, at least for colonies of Pseudomonas fluorescens on TSA and GSA. 


\section{REFERENCES}

BATES, P. K. (1928). Factors Affecting the Growth of Surface Colonies of Bacteria. Ph.D. Thesis, Massachusetts Institute of Technology.

COOPER, A. L., DeAN, A. C. R. \& Hinshelwood, C. (I968). Factors affecting the growth of bacterial colonies on agar plates. Proceedings of the Royal Society B I7I, I75-I99.

Egunov, M. A. (1915). The cell, its growth and reproduction. Investigations on the physical microbiology, chiefly in its application to the lactic acid bacillus. Trudy Vologodskovo Molotchno Instituta I, I 5-94.

EMERSON, S. (1950). The growth phase of Neurospora corresponding to the logarithmic phase in unicellular organisms. Journal of Bacteriology 60, $22 \mathrm{I}-223$.

HaINES, R. B. (1932). The influence of temperature on the rate of growth of saprophytic actinomycetes. Journal of Experimental Biology 9, 45-60.

Monod, J. (1949). The growth of bacterial cultures. Annual Review of Microbiology 3, 371-394.

PAINTER, P. R. \& MARR, A. G. (1968). Mathematics of microbial populations. Annual Review of Microbiology 22, 519-548.

Palumbo, S. A., Rieck, V. T. \& Witter, L. D. (1964). Growth parameters of surface colonies of bacteria. Bacteriological Proceedings G 106.

PIRT, S. J. (1967). A kinetic study of the mode of growth of surface colonies of bacteria and fungi. Journal of General Microbiology 47, 181-197.

SinClaiR, N. A. \& Stokes, J. L. (1962). Factors which control maximal growth of bacteria. Journal of Bacteriology 83, II 47 -II 54 .

Stokes, J. L. \& BAYNe, H. G. (1957). Growth rates of Salmonella colonies. Journal of Bacteriology 74, 200-206.

TrincI, A. P. J. (1969). A kinetic study of the growth of Aspergillus nidulans and other fungi. Journal of General Microbiology 57, 11-24. 